\title{
Coherent Control of Vacuum Squeezing in the Gravitational-Wave Detection Band
}

\author{
Henning Vahlbruch, Simon Chelkowski, Boris Hage, Alexander Franzen, Karsten Danzmann, and Roman Schnabel \\ Max-Planck-Institut für Gravitationsphysik (Albert-Einstein-Institut) and Institut für Gravitationsphysik, Universität Hannover, \\ Callinstraße 38, 30167 Hannover, Germany \\ (Received 5 April 2006; published 6 July 2006)
}

\begin{abstract}
We propose and demonstrate a coherent control scheme for stable phase locking of squeezed vacuum fields. We focus on sideband fields at frequencies from $10 \mathrm{~Hz}$ to $10 \mathrm{kHz}$, which is a frequency regime of particular interest in gravitational-wave detection and for which conventional control schemes have failed so far. A vacuum field with broadband squeezing covering this entire band was produced using optical parametric oscillation and characterized with balanced homodyne detection. The system was stably controlled over long periods utilizing two coherent but frequency shifted control fields. In order to demonstrate the performance of our setup the squeezed field was used for a nonclassical sensitivity improvement of a Michelson interferometer at audio frequencies.
\end{abstract}

It was first proposed by Caves [1] that injected squeezed states may be used to improve the sensitivity of laser interferometers and might therefore contribute to the challenging effort of direct observation of gravitational waves [2]. The goal of that proposal was the reduction of the measurement's shot noise. Later Unruh [3] has found that squeezed light can be used to correlate interferometer shot noise and radiation pressure noise thereby breaking the socalled standard quantum limit and allowing for a quantum nondemolition measurement on the mirror test mass position, for an overview we refer to Ref. [4]. Harms et al. [5] have shown that advanced interferometer recycling techniques [6] that also aim for an improvement of the signalto-shot-noise ratio are fully compatible with squeezed field injection. Gravitational-wave detectors require squeezing in their detection band from about $10 \mathrm{~Hz}$ to $10 \mathrm{kHz}$. The majority of current squeezing experiments, however, have been performed in the $\mathrm{MHz}$ regime. Furthermore the orientation of the squeezing ellipse needs to be designed for every sideband frequency. The transformation from frequency independent squeezing to optimized frequency dependent squeezing can be performed by optical filter cavities as proposed in [4] and demonstrated in [7] for $\mathrm{MHz}$ frequencies. Also in the $\mathrm{MHz}$ regime, the combination of squeezed field injection and recycling techniques has been demonstrated $[8,9]$. Squeezing at audio frequencies has been demonstrated recently for the first time $[10,11]$. However, the phase of the squeezed vacuum could not be controlled by a coherent field.

Controlling squeezed vacuum fields is the basic problem for squeezed field applications in gravitational wave $(\mathrm{GW})$ detectors. Common control schemes rely on the injection of a weak, phase modulated seed field at the carrier frequency into the optical parametric oscillator (OPO) thereby turning the device into an optical parametric amplifier. It has been shown that even lowest carrier powers introduce large amounts of classical laser noise at audio frequencies and squeezing can no longer be achieved [10].
On the other hand phase modulation sidebands are not present in a pure vacuum field. For this reason in $[10,11]$ a coherent control field for locking the squeezed quadrature angle to a local oscillator could not be created. The quadrature angle was locked instead using so-called noise locking whose stability was found to be significantly less than what can be achieved with coherent modulation locking [12] as used in GW interferometers.

In this Letter we report on the demonstration of a coherent control scheme for stable phase locking of squeezed vacuum fields. The scheme was used to produce broadband squeezing at audio and subaudio frequencies covering the complete detection band of ground based GW detectors.

The general purpose of an interferometer is to transform an optical phase modulation signal into an amplitude modulation which can be measured by a single photodiode or by a balanced homodyne detector. The observable is described by the time-dependent quadrature operator $\hat{q}_{\theta}(\Omega, \Delta \Omega, t)$ where $\Omega$ denotes the modulation sideband frequency, $\Delta \Omega$ the detection resolution bandwidth (RBW), and $\theta$ the quadrature angle. The amplitude quadrature $(\theta=$ 0 ) is usually denoted by subscript 1 and phase quadrature $(\theta=\pi / 2)$ by subscript 2 [7]. For the vacuum state the variances of quadrature operators $\left(\Delta^{2} \hat{q}_{\theta}\right)$ are normalized to unity and, generally, Heisenberg's uncertainty principle sets a lower bound for the product of the two variances of noncommuting pairs of quadrature operators, e.g., $\Delta^{2} \hat{q}_{1}(\Omega, \Delta \Omega, t) \Delta^{2} \hat{q}_{2}(\Omega, \Delta \Omega, t) \geq 1$. If an ideal phasesensitive amplifier such as a lossless OPO acts on the vacuum state the equality still holds but one quadrature variance is squeezed. For a nonclassical improvement of an interferometer's signal-to-noise ratio the angle of the squeezed quadrature then needs to be aligned to the modulation signal of interest. In realistic situations the degree of squeezing is decreased by optical losses thereby mixing the squeezed state with a vacuum, as well as by noisy classical modulation fields that beat with the same local oscillator, 
and also by acoustomechanical disturbances during the squeezed field generation and during its measurement [10].

Noisy modulation fields from the laser source can be completely removed if the squeezed light source is just seeded by a pure vacuum field. To prevent any contamination of the squeezed field, an appropriate control scheme may solely use some additional fields that do not interfere with the squeezed mode. If nevertheless such control fields are coherent with the squeezed mode full control capability is possible. The length of the OPO might be controlled by using phase modulation sidebands on a different (frequency shifted) spatial mode or polarization mode. In the experiment presented here, we used the latter. However, control of the quadrature angle in respect to a homodyning local oscillator or an interferometer is more challenging. Here we propose to use another control field that does sense the OPO nonlinearity but is frequency detuned against the vacuum squeezed mode. When coupled into the OPO the parametric gain $g$ turns the single sideband field into the following field with amplified and deamplified quadratures, respectively:

$$
E(t) \approx e^{-i \omega_{0} t} \alpha_{\Omega}[\sqrt{g} \cos (\Omega t)+i \sin (\Omega t) / \sqrt{g}]+\text { c.c., }
$$

where $\alpha_{\Omega}$ is the complex amplitude of the single sideband field with carrier detuning $\Omega$ and with phase set to zero. When $E(t)$ is directly detected with a photo diode and demodulated at $2 \Omega$ an error signal for the quadrature angle can be produced that allows coherent locking of the second harmonic pump field with respect to the control field.
Furthermore, when $E(t)$ is overlapped with a local oscillator at carrier frequency $\omega_{0}$ and the photo current is demodulated at $\Omega$ another error signal can be derived to lock the control field to the local oscillator (LO) thereby locking the squeezed vacuum field to the LO.

A schematic of the experiment is shown in Fig. 1. The main laser source (laser 1) was a monolithic nonplanar $\mathrm{Nd}$ :YAG ring laser of $2 \mathrm{~W}$ single mode output power at $1064 \mathrm{~nm}$. Approximately $1.4 \mathrm{~W}$ was used to pump a second harmonic generation (SHG) cavity. The design of the SHG cavity was similar to the one of the OPO cavity described below but with an outcoupling mirror reflectivity of $R=$ 92\%. A more detailed description of our SHG can be found in [7]. About $60 \mathrm{~mW}$ of the second harmonic field was effectively used to pump the squeezed light source. The OPO was an hemilithic optical resonator consisting of a $7 \%$ doped $\mathrm{MgO}: \mathrm{LiNbO}_{3}$ crystal and an piezo mounted output coupler. The power reflectivity of the coupling mirror was $95.6 \%$ at $1064 \mathrm{~nm}$ and $20 \%$ at $532 \mathrm{~nm}$. The curved back surface of the crystal had a high reflection coating $(R=99.96 \%)$ for both wavelengths. The cavity's free spectral range was $3.8 \mathrm{GHz}$. Orientation of the nonlinear crystal was such that $s$-polarized fields could sense parametric gain. For OPO length control we used a phase modulated $p$-polarized field which was supplied by another monolithic nonplanar Nd:YAG ring laser (laser 2). We determined a frequency shift of about $1.4 \mathrm{GHz}$ between the two polarization modes for simultaneous resonance inside the OPO. The frequency offset was controlled via
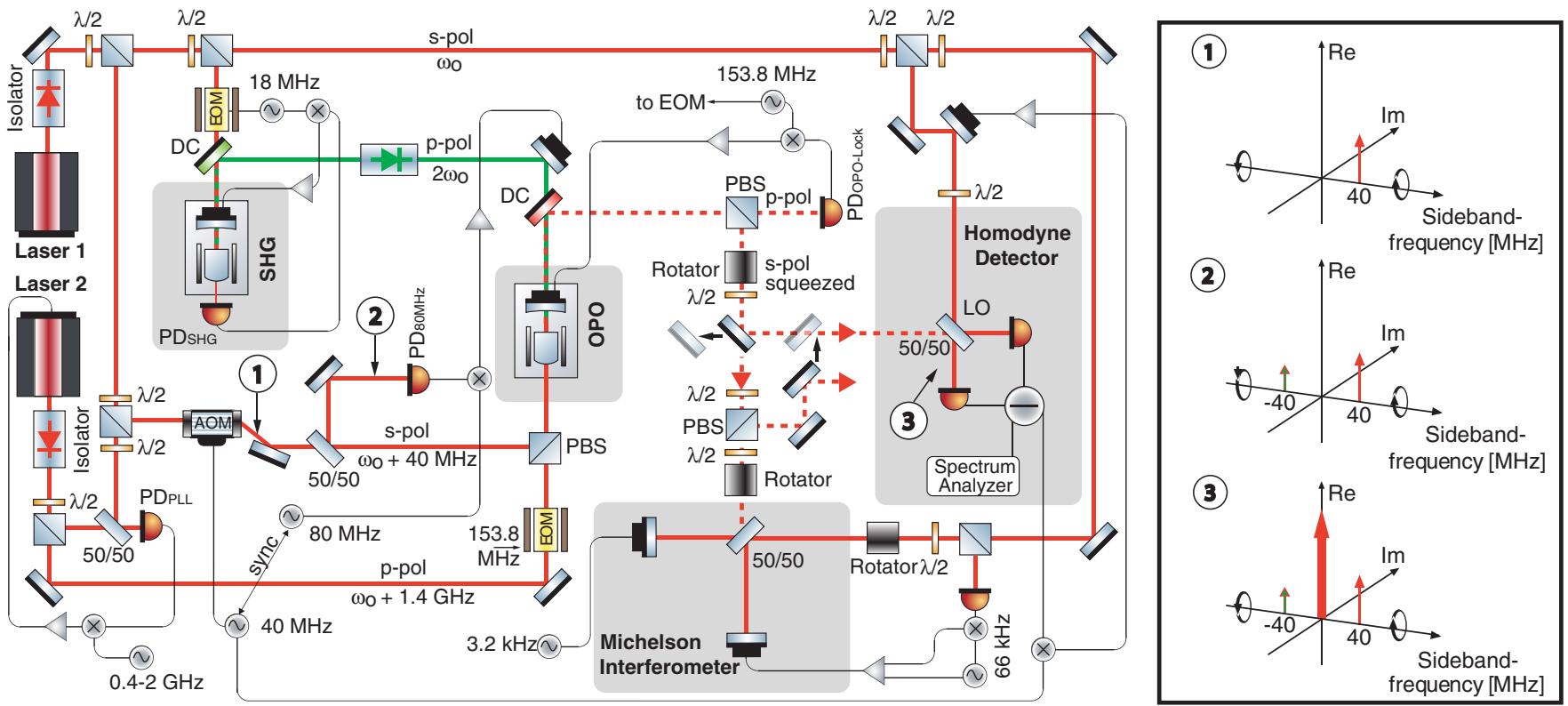

FIG. 1 (color online). Left: Schematic of the experiment. Generation and full coherent control of a broadband squeezed vacuum field at $1064 \mathrm{~nm}$ was achieved utilizing two independent but phase locked laser sources. Laser 1 provided the main carrier frequency of homodyning local oscillator and Michelson interferometer $\left(\omega_{0}\right)$. It also provided a frequency shifted control field utilizing an AOM and the OPO pump field after SHG. Laser 2 provided another frequency shifted control field. PBS, dichroic mirror (DC), LO, photodiode (PD), electro-optical modulator (EOM), PZT (ם). Right: Complex optical field amplitudes at three different locations in the experiment. 
a high bandwidth phase locking loop. The $p$-polarized locking field was injected through the crystal's back surface and spatially separated from the $s$-polarized squeezed vacuum with a polarizing beam splitter (PBS) behind the OPO. An error signal for the OPO cavity length could be generated by demodulating the detected $p$-polarized beam at a sideband frequency of $153.8 \mathrm{MHz}$ without introducing any significant loss on the squeezed field. We measured the loss to be less than $0.1 \%$ given by the PBS's power reflectivity for $s$-polarized light of $99.9 \%$. We point out that the $p$-polarized control field did not add any significant noise to the squeezed mode, given the low residual power contribution after the PBS and the high frequency offset.

Phase control with respect to a local oscillator at the fundamental frequency $\omega_{0}$ is required for both characterization and application of audio-band squeezed vacuum fields. Following our proposal described above we utilized another coherent but frequency shifted control field. Detuned by $40 \mathrm{MHz}$ with respect to the main carrier frequency $\left(\omega_{0}\right.$, laser 1$)$ by an acousto-optical modulator (AOM) this $s$-polarized infrared field $(440 \mu \mathrm{W})$ was also injected into the OPO cavity where its quadratures are parametrically amplified and deamplified which generates a sideband at $-40 \mathrm{MHz}$. This mechanism derived an error signal for controlling the phase relation between the $40 \mathrm{MHz}$ control field and the green pump. The error signal could be obtained by detecting the control field backreflected from the OPO and demodulating the photo current at twice the beat frequency $(80 \mathrm{MHz})$ as illustrated in the sideband scheme in Fig. 1. By feeding back the error signal to a piezoelectric transducer (PZT)-mounted mirror in the path of the green pump field stable phase control was realized. A separate locking loop was required to control the phase between the squeezed vacuum field, given by the phase of the green pump, and the LO. The appropriate error signal was derived at the homodyne detector, described in [7]. By taking the difference of both homodyne photodiodes a demodulation at a frequency of $40 \mathrm{MHz}$ provides an error signal which again was fed back to a phase shifting
PZT-mounted mirror. Generated and detected fields at three locations of the experiment are shown in the box in Fig. 1.

Observation and characterization of squeezed fields at audio frequencies is usually performed with balanced homodyne detection as it is done in the $\mathrm{MHz}$ regime. However, at low frequencies measurement time increases and stable control of all the field's degrees of freedom is essential. In addition homodyne detection at audio frequencies requires a much greater classical noise suppression. Figure 2 (left) verifies that our detector was indeed quantum noise limited over the full spectrum from $10 \mathrm{~Hz}$ to $10 \mathrm{kHz}$. For these measurements the (squeezed) signal input in Fig. 1 was blocked. A change of local oscillator power by a factor of 2 resulted in a change of measured quadrature noise variance of the same factor, whereas classical noise from the local oscillator would scale with the squared factor. The nominal local oscillator power used was $88 \mu \mathrm{W}$. In all measured spectra shown here the electronic noise of the detection system was at least $7 \mathrm{~dB}$ below shot noise and has been subtracted from all data. The rollup at low frequencies clearly corresponds to the transfer function of our homodyne detector. This transfer function was independently measured by modulating the local oscillator power at frequencies swept over the spectrum of interest.

The OPO squeezing spectrum from $10 \mathrm{~Hz}-10 \mathrm{kHz}$ is shown in Fig. 2 (right). Trace (a) shows the shot-noise limit of the homodyne detection system whereas the squeezed quantum noise is shown in trace (b). The measurement time was approximately 1.5 hours during which the setup was stably controlled in all degrees of freedom. Figure 3 clearly shows that $4 \mathrm{~dB}$ squeezing has been achieved over the complete detection band of ground based GW interferometers. For this plot the traces from Fig. 2 have been divided by the measured shot noise. The almost white nonclassical noise suppression has continued up to the OPO cavity bandwidth of $27 \mathrm{MHz}$. At frequencies below $10 \mathrm{~Hz}$ the squeezing measurements suffered from nonsta-

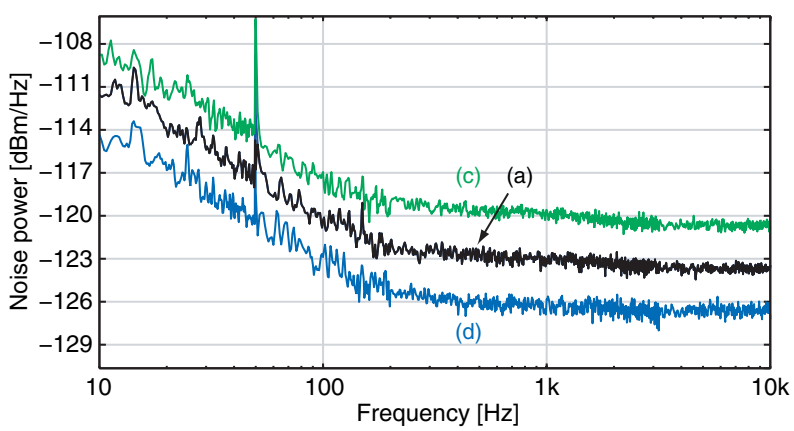

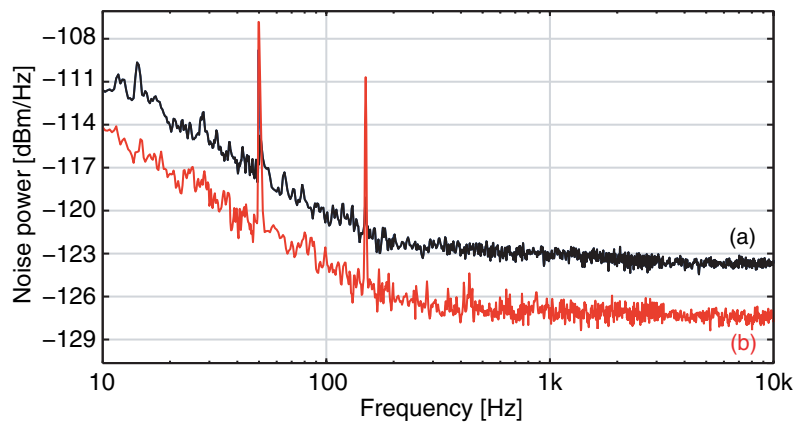

FIG. 2 (color online). Measured quantum noise spectra. Left: Shot noise for three different LO powers, (a) 88, (c) 176, and (d) $44 \mu \mathrm{W}$. Right: (a) Shot noise and (b) squeezed noise with $88 \mu \mathrm{W}$ LO power. All traces are pieced together from five FFT frequency windows: $10-50 \mathrm{~Hz}, 50-200 \mathrm{~Hz}, 200-800 \mathrm{~Hz}, 800-3.2 \mathrm{kHz}$, and $3.2-10 \mathrm{kHz}$. Each point is the averaged rms value of 100 , 100, 400, 400, and 800 measurements in the respective ranges. The RBWs of the five windows were $250 \mathrm{mHz}, 1,2,4$, and $16 \mathrm{~Hz}$, respectively. Linearity of the detection system was carefully checked in a separate measurement. 


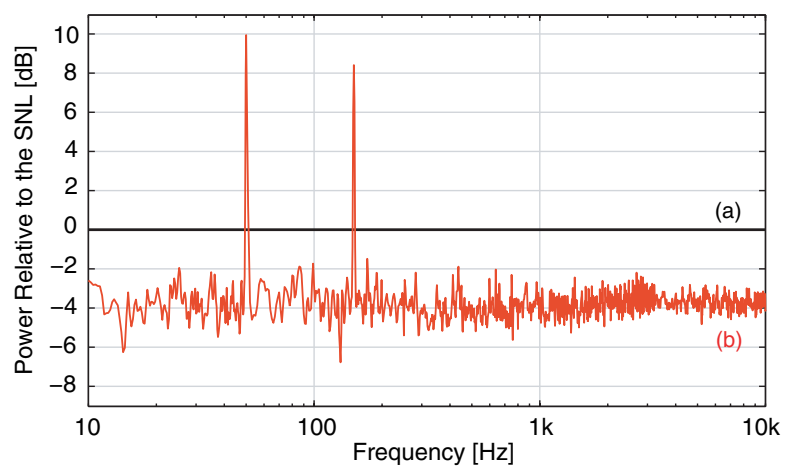

FIG. 3 (color online). Squeezed quantum noise plotted relatively to the shot-noise level. Nonclassical noise suppression of about $4 \mathrm{~dB}$ with an almost white spectrum is demonstrated. The measurement corresponds to Fig. 2 (right).

tionary noise and the degree of squeezing could not be calibrated. Peaks at 50 and $150 \mathrm{~Hz}$ were picked from stray fields and are due to the electric mains.

In addition to demonstrating long term stable generation and observation of squeezed quantum noise in the gravitational-wave band we applied the squeezed field for nonclassical sensitivity improvement of a Michelson interferometer (MI) at audio frequencies. The squeezed field was injected into the interferometer's dark port via a Faraday rotator to replace the ordinary vacuum. Since the MI was locked onto a dark fringe, the squeezed quantum noise was reflected and interfered with the interferometer signal on the homodyne detector. A fringe visibility of $99.9 \%$ at the 50/50 MI beam splitter was achieved. The MI had an arm length of $40 \mathrm{~mm}$ and was composed of two PZT-mounted flat end mirrors $(R=99.92 \%)$. Interferometer laser power was $1.5 \mu \mathrm{W}$. The error signal for locking the MI dark port was generated by longitudinal modulation of one end mirror at a frequency of $66 \mathrm{kHz}$ and

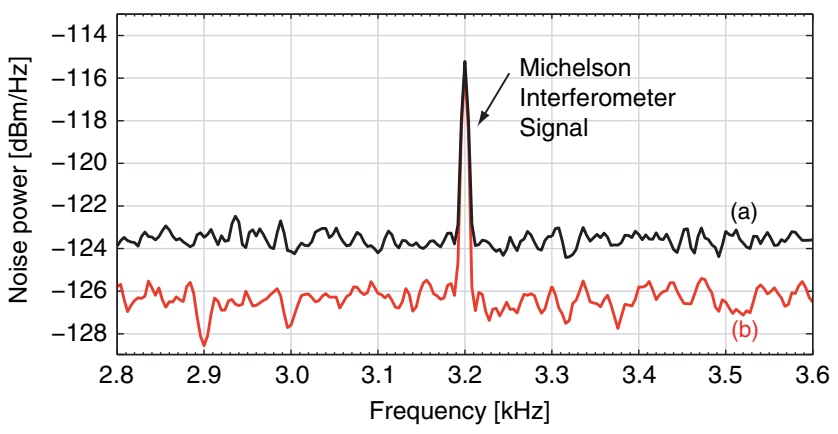

FIG. 4 (color online). Measured signal with quantum noise floors of a stably controlled Michelson interferometer without (a) and with squeezed field injection (b). The latter one shows a nonclassical signal-to-noise improvement of $3 \mathrm{~dB}$. The RBW was $4 \mathrm{~Hz}$, each point was averaged 200 times. proper demodulation of the MI bright port reflected light. The second PZT-mounted MI endmirror was modulated at $3.2 \mathrm{kHz}$ to simulate the effect of a gravitational-wave signal. The angle of the squeezed quadrature was controlled to provide a nonclassical sensitivity improvement as shown in Fig. 4. In this experiment the squeezed beam experiences an extra 5\% transmission loss from double passing the rotator and additional $7 \%$ loss due to a reduced homodyne fringe visibility (90.7\% instead of $94.3 \%)$. This additional loss degraded the observed squeezing from about $4 \mathrm{~dB}$ in Fig. 3 to then about $3 \mathrm{~dB}$.

In conclusion, we have reported on a new control scheme that allowed a stable generation, characterization, and application of squeezed fields at audio frequencies. Broadband squeezing in the full gravitational-wave detection band and a nonclassical interferometer at audio frequencies have been demonstrated for the first time. The coherent control technique reported in this Letter provides an important stepping stone for the stable application of squeezed light in GW detectors.

This work has been supported by the Deutsche Forschungsgemeinschaft and is part of Sonderforschungsbereich No. 407.

[1] C. M. Caves, Phys. Rev. D 23, 1693 (1981).

[2] K.S. Thorne, in 300 Years of Gravitation, edited by S. W. Hawking and W. Isreal (Cambridge University Press, Cambridge, England, 1987), p. 330.

[3] W. G. Unruh, in Quantum Optics, Experimental Gravitation, and Measurement Theory, edited by P. Meystre and M. O. Scully (Plenum, New York, 1983), p. 647.

[4] H. J. Kimble, Y. Levin, A. B. Matsko, K. S. Thorne, and S. P. Vyatchanin, Phys. Rev. D 65, 022002 (2002).

[5] J. Harms, Y. Chen, S. Chelkowski, A. Franzen, H. Vahlbruch, K. Danzmann, and R. Schnabel, Phys. Rev. D 68, 042001 (2003).

[6] B. J. Meers, Phys. Rev. D 38, 2317 (1988).

[7] S. Chelkowski, H. Vahlbruch, B. Hage, A. Franzen, N. Lastzka, K. Danzmann, and R. Schnabel, Phys. Rev. A 71, 013806 (2005).

[8] K. McKenzie, D. A. Shaddock, D. E. McClelland, B.C. Buchler, and P. K. Lam, Phys. Rev. Lett. 88, 231102 (2002).

[9] H. Vahlbruch, S. Chelkowski, B. Hage, A. Franzen, K. Danzmann, and R. Schnabel, Phys. Rev. Lett. 95, 211102 (2005).

[10] K. McKenzie, N. Grosse, W. P. Bowen, S. E. Whitcomb, M. B. Gray, D. E. McClelland, and P. K. Lam, Phys. Rev. Lett. 93, 161105 (2004).

[11] K. McKenzie, M. B. Gray, S. Goßler, P. K. Lam, and D. E. McClelland, Classical Quantum Gravity 23, S245 (2006).

[12] K. McKenzie, E. E. Mikhailov, K. Goda, P. K. Lam, N. Grosse, M. B. Gray, N. Mavalvala, and D.E. McClelland, J. Opt. B 7, S421 (2005). 